Itinéraires Itinéraires

Littérature, textes, cultures

2018-2 et $3 \mid 2019$

Les imaginaires de la traduction

Traduction et hétérolinguisme : une étude comparative de trois traductions de Pas pleurer de Lydie Salvayre

Translation and Heterolingualism: A Comparative Study of Three Translations of Lydie Salvayre's Pas pleurer

Marianne Braux

OpenEdition

Journals

Édition électronique

URL : http://journals.openedition.org/itineraires/4655

DOI : 10.4000/itineraires.4655

ISSN : 2427-920X

Éditeur

Pléiade

Référence électronique

Marianne Braux, «Traduction et hétérolinguisme : une étude comparative de trois traductions de Pas pleurer de Lydie Salvayre », Itinéraires [En ligne], 2018-2 et 3 | 2019, mis en ligne le 20 février 2019, consulté le 17 juin 2019. URL : http://journals.openedition.org/itineraires/4655 ; DOI : 10.4000/ itineraires. 4655

Ce document a été généré automatiquement le 17 juin 2019

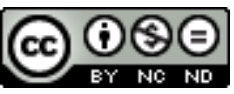

Itinéraires est mis à disposition selon les termes de la licence Creative Commons Attribution - Pas d'Utilisation Commerciale - Pas de Modification 4.0 International. 


\section{Traduction et hétérolinguisme : une étude comparative de trois traductions de Pas pleurer de Lydie Salvayre}

Translation and Heterolingualism: A Comparative Study of Three Translations of Lydie Salvayre's Pas pleurer

\section{Marianne Braux}

1 Dans Des tours de Babel, Jacques Derrida notait « une des limites des théories de la traduction: elles traitent trop souvent des passages d'une langue à l'autre et ne considèrent pas assez la possibilité pour des langues d'être impliquées à plus de deux dans un texte ${ }^{1}$ ». Suite à quoi il se demandait : «Comment traduire un texte écrit en plusieurs langues? Comment rendre l'effet de pluralité ? Et si l'on traduit en plusieurs langues, appellera-t-on cela traduire?» (Derrida 1987: 208). Comme l'indique le titre de cet article, on a choisi pour qualifier le phénomène envisagé par Derrida et aujourd'hui relativement répandu, le terme d'«hétérolinguisme» désignant dans sa définition originale « la présence, dans un texte, d'idiomes étrangers, sous quelque forme que ce soit, aussi bien que de variétés de la langue principale ${ }^{2}$ " (Grutman 1997: 37). Ce terme, introduit en France par Myriam Suchet dans sa thèse de doctorat L'Imaginaire hétérolingue (2014), présente deux avantages qui permettront d'articuler la problématique et la méthodologie du présent travail.

2 Tout d'abord, le terme d'hétérolinguisme, en impliquant le principe d'altérité, permet de cerner une caractéristique importante des textes en question: de manière générale et dans le genre romanesque en particulier, la pluralité linguistique d'un texte écrit en plusieurs langues tient à la coexistence d'une langue donnée et d'une ou plusieurs langue (s) autre(s) dont l'apparition dans le texte implique, d'un point de vue énonciatif, une mise en retrait momentanée du narrateur, une sorte de refus de traduire ce qui serait étranger à la langue principale. Ce qui explique que les romans hétérolingues comportent souvent 
un paratexte visant à combler les lacunes linguistiques du lecteur. On perçoit aussitôt l'enjeu du phénomène pour la traduction, que Derrida avait commencé de soulever : une traduction en plusieurs langues ira toujours, d'une certaine manière, à l'encontre de la traduction, dans la mesure où le traducteur y aura régulièrement suspendu son activité au profit d'une langue tierce, susceptible d'occasionner une mésentente avec le lectoratcible. Alors que pour exister en tant que telle, et avant toute littérature, la traduction est précisément censée exclure le Tiers pour s'entendre avec son destinataire, a priori monolingue. Dans la traduction hétérolingue, le vieil adage « traduttore, traditore » devient incontestable, dans la mesure où pour traduire le texte, le traducteur n'aura pas nécessairement traduit toute la langue du texte, ratant dès lors la cible de son travail. Quelle que soit la disposition du lecteur réel, seront nécessairement trahies l'attente et la confiance qu'il place dans la traduction de recouvrir l'altérité linguistique qui le sépare d'un texte auquel il n'a pas accès, faute de langue commune. C'est ainsi que l'on peut entendre le problème posé par Derrida qui, en formulant ses questions, indiquait de manière originale le paradoxe bien connu de la traduction, à la fois « ouverture, dialogue, métissage, décentrement ", disait Berman (1995 : 16), et assimilation de l'autre pour un autre. Un paradoxe que le cas-limite de la traduction hétérolingue permet d'apprécier à nouveaux frais.

Le second avantage de la notion d'hétérolinguisme est que, en mettant l'accent sur la textualité ( dans un texte») du phénomène contrairement aux termes de pluri ou multilinguisme qui sont aussi des compétences, il permet d'entreprendre une analyse du discours où les conséquences de cette trahison pourront être recherchées en vue de définir l'imaginaire linguistique du sujet énonciateur, compris selon la définition de Anne-Marie Houdebine-Gravaud comme le « rapport du sujet à la langue, la sienne et celle de la communauté qui l'intègre comme sujet parlant-sujet social ou dans laquelle il désire être intégré, par laquelle il désire être identifié par et dans sa parole » et « dont témoignent ses reprises (les siennes et celles d'autrui)» (Houdebine-Gravaud 2002: 10 et 14). Dans le cas présent, cet imaginaire concernera plus précisément le rapport au langage par lequel le traducteur désire être reconnu comme tel dans son texte et par sa langue cible, soit, sa propre conception de "la tâche du traducteur ». Un imaginaire que l'on requalifiera donc pour l'occasion de " langagier » étant donné qu'il émane de la rencontre de plusieurs langues, et que l'on se bornera à interpréter à partir de ce que les traductions ajoutent ou au contraire se gardent d'ajouter au texte original - leurs "reprises »- de manière à ne pas se laisser tromper par les intentions, aussi sincères soient-elles, du seul discours énoncé, auquel on posera la question proposée par Michel Foucault : « comment se fait-il que tel énoncé soit apparu et nul autre [ou rien] à sa place?» (Foucault 1969: 39).

4 Le corpus choisi comporte les traductions italienne, allemande et anglaise du roman hétérolingue Pas pleurer de Lydie Salvayre (2014) : Non piangere (2016), Weine nicht (2016) et Cry, Mother Spain (2016). On y relèvera et comparera les choix de traduction paratextuels et intratextuels ${ }^{3}$ de l'hétérolinguisme afin d'analyser, au niveau de l'objet-livre, comment le traducteur appréhende la tierce langue qu'elle a pris la liberté d'exposer au lecteur, au même titre que la narratrice dans le roman original. Il s'agira de décrire l'imaginaire langagier des textes en observant comment ils assument la rupture du contrat linguistique sur lequel repose la traduction, en faisant apparaitre la place qu'ils laissent au lecteur, peut-être à l'insu de leur auteur. La définition de Houdebine le dit très clairement: l'imaginaire linguistique est affaire d'image sociale de soi et ne peut se 
comprendre sans la question du désir de reconnaissance du sujet parlant par son interlocuteur, au-delà de ce qu'il peut en dire et en savoir lui-même, si l'on fait confiance aux leçons de la psychanalyse sur lesquelles s'appuie en partie la chercheuse. On ne prendra donc pas le lecteur comme une donnée acquise des textes et existant hors d'eux mais comme une figure construite dans et par le discours traducteur que celui-ci propose ou, on le verra, retire au lecteur réel. Selon Benveniste (1966:260), «le langage n'est possible que parce que chaque locuteur se pose comme sujet » et la subjectivité implique une reconnaissance mutuelle ( je pose une autre personne, celle qui devient mon écho auquel je dis tu et qui me dit $t u »)$; on essaiera donc de voir si les textes comportent un tu et lequel. On explorera ainsi, par le biais d'une problématique de plus en plus présente dans les études littéraires et traductologiques - l'hétérolinguisme - la dimension intersubjective de la traduction, occupée comme toute activité de communication à désirer le désir de son lecteur.

Il va sans dire que derrière le mot «traducteur », on entendra ici non pas l'individu mais l'instance traductrice dans laquelle intervient une myriade d'autres individus plus ou moins anonymes et en particulier, une machinerie éditoriale dont les intérêts ne sont pas toujours ceux de la littérature. Aussi, en parlant d'imaginaire du traducteur, faudra-t-il comprendre celui du texte traduit comme entité autonome produisant des valeurs dont l'énonciateur ne doit être ni nécessairement conscient pour qu'elles opèrent ni, par conséquent, tenu pour seul et unique responsable. D'autant moins que ce n'est pas dans la lecture comparée que ces valeurs deviennent réellement identifiables.

\section{Pas pleurer}

6 Pas pleurer porte sur les débuts de la guerre civile d'Espagne. Le récit est pris en charge par une narratrice, fille de réfugiés républicains espagnols, dont le texte en français se voit régulièrement interrompu par sa langue maternelle. Maternelle au sens strict car plus de la moitié du roman s'inspire du témoignage de la mère de la narratrice, Montse, rapporté au style direct libre (sans marquage typographique) et dont la langue qualifiée de « mixte et transpyrénéenne » vient scander le récit et lui imprimer son accent, via une alternance codique entre français et espagnol à même le discours narratif :

- Exemple de l'idiolecte maternel dans le premier monologue de Montse (p. 13) : mais moi, me dit ma mère, cette phrase me rend folle, je la réceptionne comme una patada al culo [...]. Alors quand on se retrouve en la rue, je me mets à griter (moi : à crier), à crier Elle a l'air bien modeste, tu comprends ce que ça veut dire ? [...] ça veut dire que je présenterai toutes les garanties d'une perfecte idiote, que je ne rechisterai jamais contre rien, que je ne causerai aucune moleste d'aucune sorte!

- Exemple de l'alternance codique dans le discours narratif (p. 55) :

José prend la parole.

C'est la première fois de sa vie. [...]

Il est théâtral. Romantique à mourir. Un ángel caído del cielo.

Il dit Nous ne voulons plus de la putasserie des possédants qui nous font une vie de misère et empochent l'argent de nos sueurs [...]. Et nous ne nous calmerons pas avec quelques os et quelques caresses. Se acabó la miseria. La revolución no dejará nada como antes. Nuestra sensibilidad se mudará también. Vamos a dejar de ser niños. Y de creer a ciegas todo lo que se nos manda.

Tonnerre d'applaudissements.

7 Contrairement à ce que nous avons pu évoquer en introduction, Pas pleurer ne comporte aucun paratexte. Cette absence est importante car elle va dans le sens du roman qui, loin 
de tout didactisme interculturel, valorise au contraire l'intraduit en chargeant les énoncés hétérolingues d'un poids avant tout symbolique. Pour le dire brièvement, ceux-ci ne relèvent pas d'une volonté de "faire couleur locale» mais reposent plutôt sur un lâcher-prise énonciatif exprimant le lien affectif qui relie la narratrice aux discours de ses personnages, sur le mode de la phrase-affect lyotardienne où, comme dans le style indirect libre, les instances énonciatrices se trouvent confondues ${ }^{4}$. À chaque occurrence espagnole, c'est en effet la mère qui parle à et par sa fille, si bien que le lecteur se trouve momentanément exclu du champ de la communication, quand bien même il serait en mesure de comprendre ce qui est dit. Les énoncés hétérolingues font ainsi événement dans le cours du récit de l'été 1936, lequel se trouve alors dépris de sa fonction purement descriptive et référentielle. Par ailleurs, comme l'indiquent le titre grammaticalement incorrect Pas pleurer ${ }^{5}$ ainsi que les interventions doucement ironiques de la narratrice cherchant à corriger le fragnol de sa mère comme dans la première citation, l'hétérolinguisme du texte fait état d'un effort littéraire de subversion de l'ordre du discours, dont toute l'œuvre de Lydie Salvayre est exemplaire ${ }^{6}$ et qu'un paratexte aurait pu discréditer. Si l'on se fie aux commentaires méta-narratifs qui ponctuent le récit, il s'agit moins pour elle de s'ouvrir, ainsi que le lecteur, à une autre langue à partir du français pris comme point de référence que de mettre en jeu la langue commune pour la voir et la faire valoir sous un autre angle. Pour le dire avec Deleuze et Guattari (1980), Pas pleurer opère une déterritorialisation de la langue française entrainnant sa reterritorialisation, indéfiniment reconduite ${ }^{7}$, exactement comme un regard détourné nous ferait changer de position. Aussi peut-on dire que la médiation linguistique qui opère dans la narration hétérolingue n'est pas, dans Pas pleurer, une médiation interlinguistique mais intra-linguistique: comme le dit Myriam Suchet dans L'imaginaire hétérolingue ${ }^{8}$, il s'agit de rendre la langue "étrangère à elle-même» (2013: 264) en feignant d'ignorer, ici et là, le lecteur francophone.

8 Tous ces effets et enjeux de l'hétérolinguisme dans le roman expliquent sans doute que les traductions à l'étude aient tenu à le reproduire, chacune à leur manière. Mais on verra que l'inquiétude du lecteur et l'ordre du discours tendent à s'y faire plus pressants, conformément au paradoxe traductif énoncé en introduction.

\section{Le rapport instructif du paratexte}

9 Une première remarque tout d'abord sur l'appareil paratextuel des traductions. Il est intéressant de constater que les trois ont fait le choix d'inclure un paratexte plus ou moins conséquent, portant à chaque fois sur la dimension espagnole du roman, là où le livre français se passe de tout commentaire. Dans un ordre croissant, la version italienne du livre comprend un "avertissement des traductrices" dans lequel est annoncée la tonalité hispanique du texte; la traduction allemande propose dans les dernières pages du livre un long glossaire linguistique et culturel allemand-espagnol; la traduction anglaise enfin, contient en amont du texte, une note du traducteur évoquant le fragnol du personnage principal ainsi qu'une préface en anglais de l'auteur Lydie Salvayre, et en aval, un glossaire des organisations politiques et militaires espagnoles mentionnées dans le texte. Dans les trois cas, le paratexte fournit aux lectorats italo/germano/anglophones des informations qui auraient pu également intéresser le lecteur francophone mais qui, en plus de n'être pas directement averti de l'altérité linguistique du texte, se voit dans l'obligation soit d'y remédier par ses propres moyens soit d'accepter de ne pas tout 
comprendre, à moins bien sûr qu'il sache lire l'espagnol. Dans les traductions en revanche, le paratexte tantôt prépare le lecteur à cette altérité via la note du traducteur, tantôt l'en répare via le glossaire. Comme si, en somme, le travail de (re)médiation interlinguistique inhérent à la traduction en tant que pratique d'interprétation, impliquait du traducteur qu'il s'excuse ou/et se rachète de ses « écarts de langue » auprès d'un lecteur à protéger, pour ainsi dire, d'une étrang(èr)eté non choisie. Et ce quand bien même le traducteur aura ensuite travaillé à produire un texte tout aussi potentiellement déroutant, linguistiquement parlant, que le texte original.

\section{Non piangere}

10 C'est le cas de la traduction italienne qui, des trois à l'étude, est celle qui reste incontestablement le plus proche du style à la fois relâché et détaché du roman original. Certes, les traductrices préviennent le lecteur des quelques «anomalies» du texte et ce faisant, énoncent en creux le principe monologique de la traduction, mais leur avertissement est avant tout argumentatif : il ne vise pas tant à réduire la distance entre le lecteur et le texte à venir qu'à convaincre ce dernier de l'intérêt d'une telle distance. Après avoir décrit les caractéristiques du parler de Montse et leur choix de l'avoir transposé «per quanto possibile» vers l'italien, les traductrices soulignent la «valori testuali » de cette originalité du roman en mentionnant l'attention qu'a portée la critique à sa signification politique, affective et intertextuelle ${ }^{9}$ ainsi qu'à son importance dramaturgique ${ }^{10}$. En s'adressant ainsi au lecteur, les traductrices tracent en quelque sorte les limites qu'elles souhaitent donner à leur tâche d'interprète : traduire, d'accord, mais à condition de ne pas rendre le texte traduit plus lisible que le texte original. Et ce aussi bien au niveau de son hétérolinguisme qu'ailleurs, comme en témoigne la fin de la note où elles avertissent qu'il leur a paru essentiel de respecter le choix de l'auteur d'omettre les signes graphiques dans les dialogues que la norme prescrit habituellement ${ }^{11}$. D'où leurs choix de traduction intratextuels qui attestent d'un effort de la langue cible pour se mouler en tous lieux sur le modèle de la narration originale en y reproduisant un dialogisme linguistique et énonciatif de même nature. L'alternance codique français / espagnol par exemple, y devient une alternance italien / espagnol, sans ajouts ni retraits :

- Pas pleurer, p. 26-27:

une montre-bracelet, des meubles en acajou, qué sé yo, c'est s'en faire esclave [...] tout sera à nous et rien ne sera à nous, comprendes ? [...]

On va faire la révolution et écraser les nationaux, s'exalte José, Fuera los nacionales! Fuera! Fuera!

- Non piangere, p. 26 :

un orologio, dei mobili di mogano, qué sé yo, significa diventarne shiavo [...] tutto sarà nostro e niente ci apparterrà, comprendes ? [...]

Faremo la rivoluzione et annienteremo i nazionalisti, si esalta José, Fuera los nacionales! Fuera! Fuera!

11 Quant à l'interlangue maternelle, les traductrices l'ont transposée de sorte à maintenir le même degré d'impact de l'espagnol sur l'italien que sur le français, quitte à remanier ponctuellement le texte pour bien en rendre compte, comme dans l'exemple suivant, issu à nouveau du premier monologue de Montse :

- Pas pleurer, p. 14 : 
Alors ma mère, pour me pacifier me rappelle à voix sussurée les bénéfices considérables qui m'espèrent si je suis engagée : [...] que j'aurai une vacation tous les dimanches [...]. À ces mots, je clame : Plutôt morir !

- Non piangere, p. 16 :

Allora mia madre per cercare di apacigarmi mi ricorda con voce sussurrata i tanti vantaggi che mi esperano se verrò assunta : [...] terrò la dominica libera [...]. Davanti a quelle palabre esclamo : Meglio morte ! espagnol la citation de Cervantès en exergue du roman et d'avoir gardé le titre à l'infinitif. Bien que "non piangere » soit correct en italien, l'altération initiale du titre français est reproduite dans l'emploi de cet infinitif valant pour un impératif, non pas moins troublé et troublant pour qui veut bien l'entendre, dès lors qu'il s'agit d'une injonction qui n'est pas adressée directement: une phrase-affect. Il n'est d'ailleurs pas surprenant que l'expression soit en italien plus idiomatique que la forme impérative.

duction italienne s'inscrit ainsi dans le modèle traductologique proposé par Brian Mossop du « translator as rapporteur » (1983) : le texte traduit y est conçu comme une réénonciation directe du texte original, avec toute la liberté linguistique que celui-ci peut comporter et sans l'intention de rétablir le sens là où le texte antérieur aurait pu le fausser. En ce sens-là, l'expérience de lecture proposée au lecteur italophone se veut la plus proche possible de celle proposée au lecteur francophone, à qui il revient d'entendre ce que respectivement les traductrices de Non piangere et la narratrice de Pas pleurer ont choisi de ne pas traduire de leurs lecture et écoute respectives. On pourrait même aller jusqu'à dire que l'expérience de lecture redouble celle du texte original, dans la mesure où les occurrences espagnoles dans le texte traduit produisent les événements discursifs du texte original une deuxième fois, y laissant entendre, non plus seulement la mère s'adressant à la narratrice ne s'adressant plus du même coup au lecteur, mais la mère s'adressant à la narratrice ne s'adressant plus aux lectrices-traductrices ne s'adressant plus non plus à leur lecteur. On s'excusera de la redondance bien trop lourde de l'expression, qui n'a d'autre but que de faire sentir ce que Roland Barthes (1975) appelait le " gouffre ${ }^{12}$ " de l'énonciation creusé par les traductrices italiennes qui figurent dans et par leur texte un lecteur à leur image.

\section{Weine nicht}

Il en va un peu différemment dans la version allemande du roman où la présence du glossaire en fin de livre indique d'emblée, comme on l'a vu, une volonté de remédiation absente de la version italienne et que l'on retrouve, dans une certaine mesure, dans les choix de traduction intratextuels. Bien que l'hétérolinguisme y soit reproduit sur le mode de la transposition comme dans la version italienne, le texte allemand manifeste une tendance à l'homogénéisation qui le distingue nettement de son homologue transalpin. Par exemple, les énoncés espagnols tendent à se laisser absorber par la langue cible, comme dans cette traduction du premier passage déjà illustré en italien :

- Non piangere, p. 26 :

un orologio, dei mobili di mogano, qué sé yo, significa diventarne shiavo [...] tutto

sarà nostro e niente ci apparterrà, comprendes ? [...]

Faremo la rivoluzione et annienteremo i nazionalisti, si esalta José, Fuera los

nacionales! Fuera! Fuera!

- Weine nicht, p. 21 :

Itinéraires, 2018-2 et 3| 2019 
eine Armbandurh, Mahagonimöbel, was weiß ich, dann macth man sich zu seinem Sklaven [...] wird uns alles und nicht gehören, comprendes ? [...]

Wir werden die Revolution machen und die Nationalisten niederwerfen, ereifert José. Fuera los nacionales ! Fuera! Raus !

De même, la citation en exergue du roman est cette fois citée en allemand et le titre, Weine nicht, en passant à l'impératif, répare en quelque sorte l'altération initiale en adressant / redressant l'injonction qui perd du même coup un peu de sa charge affective et idiomatique, que l'infinitif «nicht weinen» aurait un tant soit peu conservé. Tout se passe comme si la traductrice allemande cherchait à prolonger le travail de la narratrice de Pas pleurer qui dit « s'évertuer constamment à redresser le français bancal » de sa mère mais qui, en réfléchissant dans le texte son intention, parvient à prendre une certaine distance avec les normes qui constituent son imaginaire linguistique, au sens strict de Houdebine cette fois, et à imposer l'idiolecte maternel, "aussi sophistiqué qu'énigmatique », dans le système de la langue.

Ce comportement de la langue cible dans le texte allemand s'accuse également dans le traitement des dialogues dont on sait que la traduction italienne a voulu conserver les incertitudes graphiques. Le texte allemand en effet a plusieurs fois choisi d'en circonscrire la lecture, non pas en ajoutant des guillemets ou des tirets, mais en ponctuant par exemple des phrases laissées inachevées dans Pas pleurer et Non piangere pour représenter la parole coupée, comme ci-dessous :

- Pas pleurer, p. 23 :

Il dit que plus jamais l'argent ne décidera de toutes choses, que plus jamais il ne fondera les distinctions entre les êtres, et que bientôt

La mer aura un goût d'anisette, fait la mère agacée.

- Non piangere, p. 23 :

Dice che presto non sara piu il denaro a decidere le sorti del mondo e a fare la

differenza tra gli uomini, e che un giorno

Il mare sapra di anisetta, dice la madre irritata.

- Weine nicht, p. 18 :

Er sagt, [...] dass es nie wieder die Unterschiede zwischen den Menschen bergünden wird und dass bald.

Das Meer nach Anisette schmecken wird, ruft die Mutter ärgerlich.

Ou bien en reformulant une bribe de dialogue de sorte à bien en identifier les locuteurs, comme ici :

- Pas pleurer, p. 76 :

Qu'en penses-tu?

Formidable!

- Weine nicht, p. 65 :

Was hältst du von meiner Idee, Juan?

Wunderbar!

17 Aussi anodines ces transformations puissent-elles paraitre, elles sont, au regard des autres choix relevés, tout à fait révélatrices du comportement global de la langue cible qui, emportée par son travail d'interprétation linguistique du texte, en atténue au passage quelques obscurités : l'hétérolinguisme y est tempéré à l'intérieur du texte et après coup dans le glossaire, de même qu'y sont fixés certains flottements de sens dans le discours rapporté. Par ailleurs, l'enjeu de ces transformations prend une réelle importance lorsque l'on découvre par exemple dans la version anglaise une tout autre interprétation du dernier dialogue cité, attribué non plus aux personnages du roman qui apparaissent avant le dialogue (Juan et José), mais à la narratrice et à sa mère : « What do 
you think of that, eh? (my mother asks me) / Pretty impressive» (p. 66, c'est le traducteur qui souligne en italique).

\section{Cry, Mother Spain}

Dans la version anglaise du roman, cette volonté de la traduction de communiquer au lecteur le sens supposé du texte original prend un statut particulier en s'exprimant à tous les niveaux du livre dans ce qui apparaît comme un véritable effort de clarification, soutenu par le traditionnel imaginaire religieux de la «révélation » où la traduction se veut exégétique et au service d'un sens posé comme originel et transcendantal. Tout d'abord, le traducteur précise dès la « Translator's note » et par le biais du remerciement, sa dette à l'égard de Lydie Salvayre dont la « guidance » et le "precious insight» lui ont permis de comprendre le roman dans toute sa « complexity » et de réaliser sa traduction. Ce faisant, il place immédiatement son texte sous l'autorité de l'auteur dont il se pose en strict messager, comme en témoigne d'ailleurs le choix de donner à Lydie Salvayre la parole en préface du roman. Pour reprendre le modèle de Mossop et le déplacer un peu au moyen de la dichotomie de Ducrot entre « locuteur » et « auteur empirique » (1984: 195), il s'agit moins ici de rapporter le texte en tant que discours produit par un locuteur, à l'existence par définition fictive, la narratrice, que comme discours produit par un auteur réel et responsable de son sens, à qui l'on peut se référer pour contenir les éventuelles libertés d'interprétation qu'implique la lecture et a fortiori, la traduction.

Ensuite, toujours dans la note et contrairement à la traductrice allemande qui ne s'exprime pas directement sur le sujet, le traducteur indique la présence de son glossaire en fin de livre, en expliquant qu'il vise à rendre les références culturelles espagnoles « as clear and distinct as possible ». Il marque ainsi son travail du sceau de l'intelligibilité et se présente au lecteur en éclaireur du texte original, dont la tâche est d'assurer la transmission de son contenu étranger, y compris là où ce dernier aura omis d'en informer son propre lecteur. À ce propos, la « culturation » du titre original en Cry Mother Spain est sans doute le signe le plus explicite de cette démarche, puisqu'il attire dès la couverture l'attention du lecteur sur une dimension du texte passée sous silence dans les autres versions ${ }^{13}$.

En ce qui concerne les choix de traduction intratextuels de l'hétérolinguisme, ils présentent par rapport aux autres traductions quelques originalités toutes symptomatiques de cet imaginaire de la traduction-révélation mise en scène dans le paratexte. Le fragnol de Montse tout d'abord, n'a pas été transposé vers la nouvelle langue cible comme dans les autres traductions mais ponctuellement importé dans son mélange français/espagnol à l'intérieur du texte anglais. Soucieux d'exposer au lecteur l'accent transpyrénéen de Montse étroitement lié au contexte culturel du récit, le traducteur a par exemple choisi de conserver les «ma chérie » français de la mère à sa fille (devenus « meine Liebe » et « figlia mia » en allemand et italien), ainsi qu'un certain nombre des hispanismes si caractéristiques de son fragnol, systématiquement accompagnés de leur traduction anglaise à même le discours du personnage :

- Cry Mother Spain, p. 13-14 :

So when we are in the street again, I start to shriek, to griter: "She seems quite humble"! [...]

It means don Jaime will pay me, how do you say it? clopinettes, peanuts, and I'll have to say muchísimas gracias 
I'd bandaged it up - a panadis, isn't it called? - oh, a panaris, a whitlow, is that even a word?

At these words I say: I would prefer to die, dying would be better, plutôt morir.)

Comme on le voit, ce mode d'exposition du fragnol dans l'anglais a pour conséquence de diffracter le parler hybride de Montse en une énonciation invraisemblable dans la réalité des langues parlées, qui place du même coup le lecteur dans la position d'apercevoir momentanément derrière le texte anglais le texte original. En d'autres termes, la langue cible agit dans ces passages comme un écran qui voile et dévoile le texte original et se charge de combler, dans le co-texte, les éventuels trous de sens creusés par l'apparition des énoncés originaux.

En ce qui concerne l'alternance codique, elle n'y est pas non plus reproduite comme dans Pas pleurer ou Non piangere, mais rendue à chaque fois plus accessible au lecteur anglophone au moyen de trois stratégies. La première est celle que l'on a pu observer dans le texte allemand et qui consiste à remplacer en langue cible certaines parties des énoncés espagnols :

- Pas pleurer, p. 27 :

On va faire la révolution et écraser les nationaux, s'exalte José, Fuera los nacionales! Fuera! Fuera!

- Cry Mother Spain, p. 24 :

We're going to lead the revolution and crush the Nationalists, José shouted ecstatically. Out with the Nationalists! Out! Out! Fuera!

La deuxième stratégie consiste à remanier le discours narratif de façon à mentionner l'acte de langage exprimé en espagnol, pour en suggérer le sens :

- Pas pleurer, p. 24 :

Depuis son retour de Lérima, José est intarissable et alterne sans cesse des moments où il fulmine, où il enrage, où il multiplie les coño, les joder, les puñeta et les me cago en Dios, et d'autres où sublimement il s'exalte.

- Cry Mother Spain, p. 22 :

Since returning from Lérida, José was bursting with an inexhaustible stream of ideas. Sometimes he was raging - sprinkling his sentences with swear words (coño, joder and dozens of puñetas) - other times he was simply elated.

La troisième enfin, et aussi la plus courante, consiste à apposer aux énoncés espagnols leur équivalent anglais, comme dans les interventions de Montse et comme ci-dessous encore :

- Pas pleurer, p. 26 :

[...] une montre-bracelet, des meubles en acajou, qué sé yo, c'est s'en faire esclave

- Cry Mother Spain, p. :

[...] a wrist-watch, mahogany furniture, qué sé yo ? who knows ? Anything, is to make yourself the slave of that object

Ces deux dernières stratégies sont intéressantes car elles opèrent à première vue comme dans le texte original, lorsque le français et l'espagnol se font écho dans le cours de la narration, comme dans les extraits suivants :

[José] promet à sa sœur [...] un monde juste et beau, un paraíso, il en rit de bonheur, un paradis réalisé où l'amour et le travail (p. 25)

Le dernier paseo (la dernière promenade, c'est ainsi que l'on dit). (p. 69)

Ou lorsque la narratrice précise l'effet que produisent les propos de sa mère :

El tiempo hace y deshace, un tal gusta y disgusta otro dia, hay que acostumbrarse, commente ma mère qui parle parfois comme un publicitaire. (p. 66)

Mais dans Pas pleurer, plus que des stratégies de traduction cherchant à favoriser la compréhension, ces procédés sont évidemment avant tout stylistiques - on voit bien 
d'ailleurs dans le premier exemple (paradis / paraíso) que pour le lecteur francophone, la traduction apposée n'a rien de vraiment nécessaire. De plus, ces procédés sont à mettre en regard de procédés inverses par lesquels la narratrice omet volontairement de traduire certains énoncés, inscrivant ainsi la narration dans une dynamique contrapuntique, où français et espagnol tantôt se répondent tantôt s'ignorent, cohabitant dans le texte sur le mode non pas de l'équivalence mais de la complémentarité. D'où, parfois, une différenciation activement entretenue par la narratrice. C'est par exemple le cas dans l'extrait suivant où un mot espagnol est ouvertement qualifié d'intraduisible :

Tous ces verbiages sans rapport avec la réalité risquent d'entraîner le village dans un desmadre (le mot, intraduisible, fait beaucoup d'effets sur la population paysanne). (p. 64)

Or on sait que l'intraduisibilité, comme la traduisibilité, est un toujours un choix et le signe d'une posture face à la question de la communication; ce qui compte donc est d'apprécier la tension que ce choix instaure entre l'une et l'autre langue. Une tension que le traducteur réduit inévitablement quand il choisit ici de traduire ce qu'il maintient pourtant comme "intraduisible», en expliquant par conséquent ce qui se voudrait inexplicable dans un énoncé à la limite du non-sens, mais qui reste cohérent par rapport au comportement global de la langue cible dans le texte ${ }^{14}$ :

[...] risked dragging it towards a desmadre (meaning pandemonium, chaos and excess, an untranslatable word that had a big impact on the farmers in the audience).

(p. 56-57)

Dans la typologie de Suchet, ce mode de retranscription de l'hétérolinguisme serait à situer dans la catégorie de l'« hétérolinguisme problématique », dans la mesure où le texte d'arrivée « renvoie sans cesse au texte de départ sans jamais s'établir comme texte autonome » (2014: 262). Mais comme le dit justement la chercheuse, à propos d'une autre catégorie de traductions présentant à l'inverse une grande indépendance vis-à-vis du texte source que "l'on pourrait déplorer ", cette démarche pourrait tout aussi bien être considérée, en l'absence de norme traductive officielle, comme « un des modes possibles de relation au texte source " $(2014: 262)$. On dira donc plutôt que le problème ne réside pas tant dans le fait que le texte ne devienne jamais autonome que dans le type de rapport inter-linguistique qu'il instaure, en obligeant pour ainsi dire la langue cible à marquer son territoire dans la remédiation des incongruités linguistiques provoquées par une traduction qui, parce qu'elle a choisi d'exhiber sa source, cherche en contrepartie à ne pas perdre de vue sa cible ${ }^{15}$. Le langage comme outil de communication imparable ainsi représenté, c'est la figure du lecteur qui, contre toute attente, s'effondre: un tel surmenage exégétique en effet ne peut aller qu'à l'encontre de son statut même de lecteur. Pas seulement celui qu'il tire de sa qualité de libre interprète, qui sera d'autant moins effective que le texte aura déjà comblé ses propres trous - c'est un fait qui mérite à peine d'être répété tant il a déjà été bien décrit par les théoriciens de la réception : plus le texte oriente ma lecture, moins je lis. Plus fondamentalement, c'est le statut du lecteur en tant que libre destinataire qui est mis en jeu; celui qu'il tire, avant tout procès d'interprétation, de pouvoir recevoir une parole débarrassée, dans l'écrit, de ses alibis communicationnels, pour refonder à chaque fois la possibilité du dialogue. Parole adressée donc, mais non "communicationnaire » comme dirait Salvayre, dont le roman et la traduction hétérolingue peuvent fournir, comme l'a montré Suchet dans son ouvrage, de beaux exemples. "Contrairement à la communication, l'adresse n'implique pas la réussite de la transmission du message : c'est un pur geste tendu »; lorsqu'il suit ce 
principe, le texte hétérolingue est comme une «bouteille jetée à la mer» (2014: 129). C'est la définition même de la destinerrance chez Derrida dont l'analyse du problème de l'adresse permettrait de comparer Pas pleurer à une « carte postale » interceptée alors que Cry Mother Spain serait plutôt comme une lettre en recommandé avec accusé de réception, ou un message Whatsapp. On reconnaîtra dans cette adresse sans interlocuteur la particularité du dispositif de la cure psychanalytique où, comme dans le dispositif de l'écrit, la présence-absence de l'analyste / lecteur est ce qui permet à l'analysé / écrivain de se reconnaitre dans la fonction, non plus informative, mais vocative du langage par laquelle le sujet se constitue. Comme l'écrit Jacques Lacan : «la fonction du langage n'est pas d'informer, mais d'évoquer. Ce que je cherche dans la parole, c'est la réponse de l'autre. Ce qui me constitue comme sujet, c'est ma question » (1966:299-300).

Pour en revenir à la traduction anglaise, dont il ne s'agit pas de mettre en doute les bonnes intentions, il est certain qu'elle mène à son paroxysme le paradoxe relevé en introduction. Conformément au vœu de clarification énoncé dans le paratexte, la pluralité linguistique y est, quantitativement parlant, représentée comme dans nulle autre traduction, exposant au lecteur non plus seulement deux langues mais trois. Aussi pourrait-on penser qu'elle est celle qui, trahissant doublement le lecteur monolingue, aurait le mieux traduit le texte original dont la force de conviction repose précisément sur son jeu d'altération linguistique. Mais en même temps, on se rend bien compte après examen que la traduction anglaise assume mal ce parti pris et ne parvient pas à créer un espace pour être (mal)entendue de son lecteur, lui imposant une parole-déjà-discours trop pleine pour pouvoir être remplie puisqu'elle ne pose pas de question. Tant et si bien que l'on ne saurait dire si cette traduction est d'obédience sourcière plutôt que cibliste, l'exhibition du texte source entraînant une installation de la langue-culture anglaise en ultime point de référence.

\section{Conclusion}

Aux critiques que l'on a adressées aux traductions allemande et surtout anglaise, on pourra rétorquer par un argument très simple, celui-là même que le traducteur anglais a bien voulu nous donner dans une correspondance, et qui est indiscutable : l'anglais et l'allemand, étant des langues germaniques moins proches de l'espagnol que l'italien qui est une langue romane, il est naturel que les traducteurs aient davantage remédié aux énoncés hétérolingues du roman, sous peine de produire un texte définitivement illisible. C'est vrai, et c'est pourquoi nous avons insisté au début sur l'emploi du mot traducteur comme « instance traductrice » dans laquelle il est évident que la langue cible elle-même a sa part de responsabilité, autant que la langue source d'ailleurs, lorsque les deux n'appartiennent pas à la même famille linguistique. Il n'est pas étonnant à ce propos que la traduction espagnole du roman, No llorar, soit la moins bavarde. Aucun compte à rendre ici : la tierce langue de la traduction, l'espagnol, est la même que la langue cible si bien que le texte traduit en ressort parfaitement homolingue. Ou presque, car le traducteur a trouvé un moyen original de signifier malgré tout l'hétérolinguisme initial via la mise en gras des énoncés espagnols du texte source, matérialisant leur épaisseur énonciative et la charge affective qui y est rattachée : «Es teatral. Apasionado hasta la médula. Un ángel caído del cielo. » (p. 45). Pas d'hétérolinguisme donc, mais un hétérographisme apte à faire événement dans la lecture. 
Revenons, pour terminer, aux mots de Derrida qui demandait comment « rendre l'effet de pluralité » d'un texte écrit en plusieurs langues et laissait la question en suspens. Au vu de l'importance des notions de don et de dette chez le philosophe, le mot « rendre » n'est ici pas insignifiant et l'on pourrait, au terme de cette étude, y entendre la nécessité pour le traducteur d'imiter la parole délibérément plurielle - sa liberté comme l'« effet » de sa pluralité - afin de donner au lecteur le visage que l'écrit lui doit, dès lors que la lecture met un terme, aussi provisoire soit-il, à son errance. Quant à savoir si «l'on pourra appeler cela traduire ", peut-être que la distinction entre la communication ordinaire, qui vise le sens de la langue, et la communication littéraire, qui vise le sens du texte, permettrait de le dire. D'un point de vue " ordinaire », on [le lecteur] est en droit de refuser le titre de "traduction " à une traduction hétérolingue, dans la mesure où sa pluralité linguistique signifie que le traducteur aura volontairement négligé sa cible. De ce point de vue-là, et tant que dureront les monolinguismes, une traduction hétérolingue n'en sera jamais vraiment une. D'un point de vue «littéraire » en revanche, le lecteur aura peu de chance, s'il se plaint d'une traduction inachevée, de gagner le procès car il est, en dernière instance, responsable d'avoir intercepté une parole qui ne lui était pas directement destinée, comme l'est un lecteur original et à condition, on l'a vu, que la traduction lui réserve un espace où se saisir comme tel. Alors on [le lecteur et critique] pourra même appeler cela une traduction réussie, sans ignorer l'imaginaire langagier qu'une telle assertion implique à son tour et que l'on pourrait, avec Benveniste, résumer ainsi : « bien avant de servir à communiquer, le langage sert à vivre ${ }^{16} »(1974: 217)$.

\section{BIBLIOGRAPHIE}

Barthes, Roland, 1975, Roland Barthes par Roland Barthes, Paris, Seuil.

Benveniste, Émile, 1966, « De la subjectivité dans le langage », dans Problèmes de linguistique générale, Paris, Gallimard.

Benveniste, Émile, 1974, « La forme et le sens dans le langage », dans Problèmes de linguistique générale, t. II, Paris, Gallimard.

Berman, Antoine, 1995, L'Épreuve de l'étranger, Paris, Gallimard.

Deleuze, Gilles et Guattari, Félix, 1980, Mille plateaux, Paris, Minuit.

Derrida, Jacques, 1987, « Tour de Babel », dans Psyché. L'invention de l'autre, Paris, Galilée.

Ducrot, Oswald, 1984, Le dire et le dit, Paris, Minuit.

Foucault, Michel, 1969, L’Archéologie du savoir, Paris, Gallimard.

Grutman, Rainer, 1997, Des langues qui résonnent. L'hétérolinguisme au XIX siècle québécois, Québec, Fides.

Houdebine-Gravaud, Anne-Marie, 2002, «L'imaginaire linguistique : un niveau d'analyse et un point de vue théorique », dans A.-M. Houdebine-Gravaud (dir.), L'Imaginaire linguistique, Paris, L'Harmattan, p. 9-24. 
Lacan, Jacques, 1966, Écrits, Paris, Seuil.

Lopoukhine, Juliana, 2015, « En français dans le texte. La poétique de l'inarticulé de Jean Rhys », Revue LISA/LISA e-journal, [En ligne], vol. 13, $\mathrm{n}^{\circ}$ 1, http://journals.openedition.org/lisa/8560, consulté le 20 janvier 2019.

DOI : $10.4000 /$ lisa.8560

Mossop, Brian, 1983, « The Translator as a Rapporteur: A Concept for Training and Selfimprovement », Meta, vol. 28, n 3, p. 244-278.

Suchet, Myriam, 2014, L'imaginaire hétérolingue : ce que nous apprennent les textes à la croisée des langues, Paris, Classiques Garnier.

\section{Corpus}

Salvayre, Lydie, 2014, Pas pleurer, Paris, Seuil.

Salvayre, Lydie, [2014] 2016, Non piangere, trad. Lorenza di Lella et Francesca Scala, Rome, L'asino d'oro.

Salvayre, Lydie, [2014] 2016, Weine nicht, trad. Hanna van Laak, Munich, Karl Blessing Verlag.

Salvayre, Lydie, [2014] 2016, Cry, Mother Spain, trad. Ben Faccini, Londres, Maclehose Press.

\section{NOTES}

1. C'est Derrida qui souligne.

2. C'est Grutman qui souligne.

3. Dans les exemples, les passages comparés apparaîtront en gras et les modifications ou ajouts des traducteurs seront soulignés.

4. Pour un autre exemple, plus argumenté, du lien entre affect et hétérolinguisme mobilisant le concept de "phrase-affect» de Jean-François Lyotard, voir l'excellent article de Juliana Lopoukhine « En français dans le texte. La poétique de l'inarticulé de Jean Rhys » (2015), auquel je reprends l'emploi de l'expression «faire couleur locale » pour dénoncer l'illusion référentielle que les textes se refusent à entretenir.

5. L'expression réapparaît à la fin du roman, p. 227, dans un paragraphe où elle est implicitement attribuée à Montse : «Après maintes péripéties, elle [Monste] finit par échouer dans un village du Languedoc, où elle dut apprendre une nouvelle langue (à laquelle elle fit subir un certain nombre d'outrages) et de nouvelles façons de vivre et de se comporter, pas pleurer. »

6. Pour exemple, voici un extrait d'un texte de Lydie Salvayre intitulé « Défense et illustration du fragnol » : «Car c'est [...] Une langue impure, extrêmement, et qui, mine de rien, fait entrer de l'autre, fait entrer de l'Espagne, fait entrer des espagnes, fait entrer des autrement-dire, et peutêtre, du même coup, des autrement-penser, on s'élargit, on respire. [...] Une langue qui porte en elle une part d'opacité, dans une société que le monde communicationnaire voudrait transparente comme l'eau. La transparence, aurait dit ma mère, est le cadeau de mes soucis. Bref, une langue vivante, vivante, vivante et qui me sert constamment d'exemple.» (Bienvenue! 34 auteurs pour les réfugiés, Éditions Points, 2015).

7. Il est remarquable, à cet égard, que le roman termine de manière abrupte, sur la parole hybride de Montse : «Si tu nous servais une anisette, ma chérie. Ça nous renforcerait la morale. On dit le ou la ? / On dit le. Le moral. / Une petite anisette ma Lidia. Par les temps qui galopent, c'est une précaution qui n'est pas, si j'ose dire, surnuméraire » (p. 279). 
8. Je ne dirai pas en revanche, comme M. Suchet en introduction de sa thèse, qu'il s'agit de « faire voler en éclat le mythe saussurien de "La langue une et indivisible" ", selon une expression reprise à Grutman. Cette déclaration d'intention trahit selon moi une lecture un peu hâtive du cours de Saussure, sans doute inspirée de celle que semble en faire Bakhtine, dont la critique du structuralisme saussurien en faveur d'une "translinguistique » moins systématique et plus créatrice est séduisante à bien des égards mais pas nécessairement invalidante (voir Catherine Depretto, L'Héritage de Bakhtine). De plus, une telle expression, que nous n'avons pas trouvé, soit dit en passant, dans le cours, ne rend pas justice à la grande innovation de Saussure qui n'est pas l'invention de « la langue une et indivisible » mais bien le couple opératoire « langue / parole ».

9. « valenze politiche, affettive e intertestuali ».

10. « rivelenza drammaturgica ».

11. « rispettare la scelta autoriale di omettere i segni diacritici che di norma individuano le battute di dialogo ».

12. La citation complète est la suivante: «Une bonne part de notre travail intellectuel consiste à porter la suspicion sur n'importe quel énoncé en révélant l'échelonnement de ses degrés; cet échelonnement est infini et ce gouffre ouvert à chaque mot, cette folie du langage nous l'appelons scientifiquement : l'énonciation » (Barthes 1975 : 70).

13. Et en le transformant au passage en une formule pour le moins pathétique aux antipodes de l'affect salvayrien, il dénonce assez bien le lien entre le didactisme interculturel contemporain et l'industrie de la compassion dans nos sociétés occidentales.

14. Il est d'ailleurs intéressant de comparer ce passage avec le texte allemand qui, dans un même souci d'éclairer malgré tout le sens du mot espagnol, donne sa traduction tout en relevant explicitement son insuffisance: " ein Desmadre stürzen (dass mit Chaos nur ansatzweise übersetzbare Wort übt eine grosse Wirkung auf die Bauern aus)» (p. 55).

15. Si l'on se réfère aux travaux de Houdebine, ce comportement véhiculerait la norme dite «fonctionnelle » ou « communicationnelle » du langage, laquelle «se paie parfois d'une fiction de transparence, autrement dit de ce qu'il est convenu d'appeler un cratylisme naif » (2002:20). 16. C'est Benveniste qui souligne.

\section{RÉSUMÉS}

Le cas de l'hétérolinguisme en littérature pose à la traduction la question de son bien-fondé, lorsqu'elle prend le parti du texte source de rater sa cible en exposant le lecteur à une autre langue que la sienne. Cet article examine ce problème à travers une critique comparée des traductions italienne, allemande et anglaise de Pas pleurer de Lydie Salvayre. En mobilisant la notion d' "imaginaire linguistique " (Houdebine), on procédera à l'analyse énonciative des traductions visant à observer, au niveau de l'objet-livre (texte, paratexte, édition), comment est assumée la rupture du contrat linguistique sur lequel repose le geste interprétatif de la traduction et à faire apparaître l'image idéale du lecteur construite dans et par le discours traducteur.

The case of heterolingualism in literature questions the principles of translation, when it purports to imitate the cross-language source text and misses its target, by exposing the reader to a third language. This article examines this problem through a comparative critical study of the Italian, German and English translations of Pas pleurer by the French writer Lydie Salvayre. 
Drawing on Houdebine's notion of "linguistic imaginary," the texts will be analysed from an enunciative perspective, in order to understand how the linguistic contract of translation is compromised and also to highlight the ideal image of the reader which is formed in and by the translator's discourse (text, paratext, edition).

INDEX

Mots-clés : hétérolinguisme, traduction, Salvayre (Lydie), imaginaire linguistique, lecteur Keywords : heterolingualism, translation, Salvayre (Lydie), linguistic imaginary, reader

\section{AUTEUR}

MARIANNE BRAUX

Université d'Adélaïde (Australie) 